\title{
Automatic Identification of Hard Exudates in Retinal Fundus Images using Techniques of Information Retrieval
}

\author{
Kemal Akyol \\ Karabuk University \\ Baliklarkayasi, Karabük \\ 78050, Turkey
}

\author{
Baha Şen \\ Yıldırım Beyazıt University \\ Altındağ, Ankara \\ 06030, Turkey
}

\author{
Şafak Bayir \\ Karabuk University \\ Baliklarkayasi, Karabük \\ 78050, Turkey
}

\begin{abstract}
Diabetic retinopathy, a subject of many studies in the medical image processing field since long time, is one of the major complications of diabetes mellitus and it cause blindness. In this study, we proposed a method that consist of keypoint detector-feature extraction-reduction process and classifier stages within the framework of hybrid approach for the detection of hard exudates. This method is divided into two parts: learning and querying. In the learning phase, initially we created visual dictionaries for the representation of pathological or non-pathological regions on retinal images. After, we completed modeling process with the training and testing processes. In the querying phase, keypoints and patch images are obtained with keypoint detector algorithm from new retinal images. Thus, knowledge is obtained by these patch images are classified in the final part of this phase. Experimental validation was performed on DIARETDB1 public database. The obtained results are showed us that positive effects of machine learning technique suggested by us for diagnosis of exudate.
\end{abstract}

\section{Keywords}

Hard Exudates, Information Retrieval, Keypoint Algorithm, Local Descriptors, Visual Dictionary, Classification.

\section{INTRODUCTION}

Retina is the most internal membrane of the human eye. Diseases that occur in the retina threaten our eye-sight directly. Systemic diseases in eye such as hypertension and diabetes mellitus cause some pathological changes [1]. We can provide pathological information using digital images of the fundus eye.

Diabetic Retinopathy (DR) which affects the retina's blood flow is one of the major complications of diabetes mellitus and can cause blindness among the diabetic population. Timely diagnosis and treatment of DR can reduce the risk of severe vision loss significantly. The diagnosis of DR, traced by a specialist doctor, is performed by visual analysis of retinal images being exudates $[2,3]$.

There are many works based on the detection of DR lesions. Hard exudates (HE) are one of many kind of lesions caused by DR, which appear at early disease stages.

In this study, we present an approach to detect hard exudate from retinal fundus images based on visual descriptors. Our methodology consist keypoint and local descriptor algorithms for HE identification in retinal images. The visual dictionaries are used for generating a single feature vector to be passed to the classifier that will classify patch image whether pathological or non-pathological. The experimental validation was performed on a public image database, DIARETDB1 [4].

The rest of this paper is organized as follows. In Section 2, related studies are examined. In Section 3, it is detailed the exposition of our proposal methodology. In Section 4, it is given the experimental results of applying this method. In Section 5, discussion and future studies are presented. In final section, conclusion is presented.

\section{LITERATURE REVIEW}

There are numerous investigations in automatic retina analysis with emphasis on hard exudate detection. Chen et al presented a novel method to automatically detect HEs in color retinal images. First extract HEs candidate regions by combining histogram segmentation with morphological reconstruction. Next, they defined 44 significant features for each candidate region. A supervised support vector machine is finally trained based on these features to classify the candidate regions for HEs [5]. Eadgahi and Pourreza according to their proposed method, the retinal images preprocessed optic disc and the blood vessels identified primarily and then eliminate from the image for segmentation of hard exudates in retinal color image based on morphological operation. After, they segmented them by mixture of morphological operation such as Top-hat, Bottom-hat and reconstruction operations [6]. In their study Garcia et al. detected hard exudates automatically by extracted a set of features from image regions and selected the subset that best discriminated between hard exudates and the retinal background using logistic regression [7]. In their study Guoliang Fang et al proposed an effective framework to automatically segment hard exudates in fundus images. Framework is based on a coarse-to-fine strategy. According to this, firstly getting a coarse they resulted allowed of some negative samples then eliminate the negative samples step by step [8]. In their study Hsu et al. demonstrated the role of domain knowledge in improving the accuracy and robustness of detection of hard exudates in retinal images [9]. Kayal and Banerjee proposed a method uses various image processing techniques, such as median filtering, image thresholding etc. for detect hard exudates [10]. Mishra et al introduced a novel way for detection HEs, using the Distance Learning Metric. Also they introduced a new method to remove the optic disc in the post-processing stage based on variance calculation [11]. In their study Ranamuka and Meegama proposed a technique based on morphological image processing and fuzzy logic to detect hard exudates from DR retinal images. At the initial stage, the exudates are identified using mathematical morphology. Subsequently, hard-exudates are extracted using an adaptive fuzzy logic algorithm that uses 
values in the RGB color space of retinal image to form fuzzy sets and membership functions [12]. Sánchez et al developed the detection of hard exudates in retinal images incorporating contextual information in the computer-aided detection system. The context is described by means of high-level contextual-based features based on the spatial relation with surrounding anatomical landmarks and similar lesions [13]. In another study, Sanchez et al proposed an automatic method to detect hard exudates. The algorithm is based on mixture models in order to separate hard exudates from background [14]. In their study Tjandrasa et al segmented hard exudates using mathematical morphology and achieved features. The extracted features are classified by using soft margin support vector machine [15]. In their study $\mathrm{Xu}$ and Luo presented a novel method to identify hard exudates from digital retinal images using feature combination. It is based on stationary wavelet transform and gray level co-occurrence matrix to detect hard exudates. An optimized support vector machine with Gaussian radial basis function is used as a classifier [16].

Digital data can be commonly used in healthcare studies to help the physician on determining the appropriate diagnosis and the treatment method. Akyol et al. used gabor filter kernels for detection of optic disc [17]. In particular recursively, they investigated the presence of the candidate of the optic disc and making the analysis. Kaya et al. used Watersheed Segmentation and thresholding methods to segment the Keratoconus region from the cornea [18].

\section{THE PROPOSED METHODOLOGY}

In this section, we detail essential information used in our study. Visual information is the strong and effective representation of the digital images. It is crucial to the performance of image retrieval and classification. The main objective of this study be able to perform successful analysis creating models based visual information.

\subsection{Keypoint Detector and Local Descriptors}

Keypoints are salient image patches that contain rich local information of an image. Based on keypoints information extraction as salient image patches, an image can be described as a bag of visual words and this representation has been frequently used in the classification of imagery data [19]. Images can be automatically detected using various keypoint detectors, which are surveyed in [20].

We find a set of points of interest (PoI) in such images and determine their surrounding regions to represent the visual information of a given image. It is aimed to choose scale invariant interest points for achieving a representation robust to some possible image transformations. We examined several studies, a visual dictionary was created with keypoint algorithms's local descriptors. The local information can be provided not only by using keypoint algorithm but also by the other descriptor algorithms for patch images.

After detecting keypoints, we must determine which features come from corresponding patch in images. The main idea is to quantize local descriptors into "visual words" and represent each patch image as a vector of words as a document. According to this, we represent every image in a collection using points of interest (PoI) using the Oriented FAST and Rotated BRIEF (ORB) approach. In their study Rublee et al. introduced ORB algorithm as fast and efficient [21]. After that, local features are calculated by using K-Means and DoG algorithms and their variant K-Means-PCA and DoG-PCA.
Here we use McQueen's 'kmeans' algorithm [22] and simple difference of gaussians (DoG) approximation [23] for local descriptors. The goal of use Principal Components Analysis (PCA) is eliminate to features the least influential in the classification, decreasing complexity and increasing its efficiency.

\subsection{Visual Dictionary}

The visual dictionary, composed of words, generates signatures for each image. It is a robust method using of the visual dictionary to learn and represent important features of a given anomaly even in the existence of noise such as image distortion [24]. Besides, such method is robust against differences in color background of the retinal fundus images. The visual dictionary (codebook) is created representing distinctive features of images using local features.

Based on visual dictionary, we are able to classify whether a patch image is pathological or non-pathological. After creating the dictionary, we perform the assignment of each of the training images' PoIs to the closest visual word of the dictionary with clustering technique. This step is known as quantization [25]. The analogy between patch images and words in visual dictionary provide opportunities for techniques of information retrieval that is used to solve problems in image data. In order to provide a successful classification, it is planned to apply information retrieval techniques based on the bag-of-visual-words representation.

\subsection{Classification}

Classification is the process of finding which data belongs to the variety of classes through training and observation. A model, based on classification, is trained with training data and is asked to decide which test data are belong to which class. The best sampling of data space is created the most performance.

Image classification is a well-studied topic in the field of image processing and computer vision. State-of-the-art approaches include robust features and classification algorithms. Such features can then be used by classifiers such as Random Forest (RF) algorithm to perform image classification. The goal of image classification is to predict the categories of the input image using its features.

$\mathrm{RF}$ algorithm is a very efficient and popular algorithm for classification and regression problems introduced by Breiman [26]. A random forest multi-way classifier which consists of a number of trees, with each tree grown using some form of randomization, is one of machine learning techniques. Estimates of the posterior distribution over the image classes label the leaf nodes of each tree. By sending it down every tree and aggregating the reached leaf distributions, an image is classified. It is possible to inject randomness at two points during training: in subsampling the training data so that each tree is grown using a different subset; and in selecting the node tests [27].

\subsection{Performance Measures}

The medical data is usually classified into two classes where the disease is either present or absent. The classification accuracy of the diagnosis is assessed using the sensitivity and specificity measures. Sensitivity is the percentage of abnormal finding classified as abnormal and specificity is the percentage of normal finding classified as normal. High sensitivity and specificity value indicates that better manner of the diagnostic. Sensitivity and specificity are as in the following Equation 1 [28, 29]: 
Sensitivity $(\mathrm{SN})=\mathrm{TP} / \mathrm{TP}+\mathrm{FN}$

Specificity $(\mathrm{SP})=\mathrm{TN} / \mathrm{TN}+\mathrm{FP}$

In this equality, TP is the number of exudate region found as exudate; TN is the number of normal region found as normal. $\mathrm{FP}$ is the number of normal region found as exudate and FN is the number of exudate region found as normal in our study.

\section{EXPERIMENTAL RESULTS}

We tested our approach on the publicly available DIARETDB1 color fundus image database, all of same size $(1500 \times 1152)$. Light intensity in conditions and changes in light cause difficulties extracting the information from image in RGB color space. HSV (Hue, Saturation and Value) color space is defined as hue, saturation and value distinctly from RGB color space. Therefore, light changes in HSV color space can be easily recognized and difficulties during the image processing can be removed.

In the first phase of the study, all RGB (Red, Green and Blue) fundus images are converted into HSV color space. We evaluated the methodology on testing new retinas and demonstrated the robustness and reliability of the approach performing five folds cross-validation.

In the learning phase, firstly we obtained local features from 150 pathological and 150 non-pathological patch images that determined by the specialist doctors. The local features were obtained using KMeans and DoG algorithms, their variant with reducing the PCA (K-Means-PCA, DoG-PCA). In the next step, we created two visual dictionaries or codebooks, called Codebook-1 and Codebook-2, from these feature sets for distinctive features of images with pathological or nonpathological. After creating the dictionaries, we performed the assignment of each of the training image to the closest visual word of the dictionary with K-Means clustering algorithm (quantization). In the end of quantization process, we used RF classifier in order to perform the success of the developed system. Figure 1 shows the flowchart of these steps in our methodology.

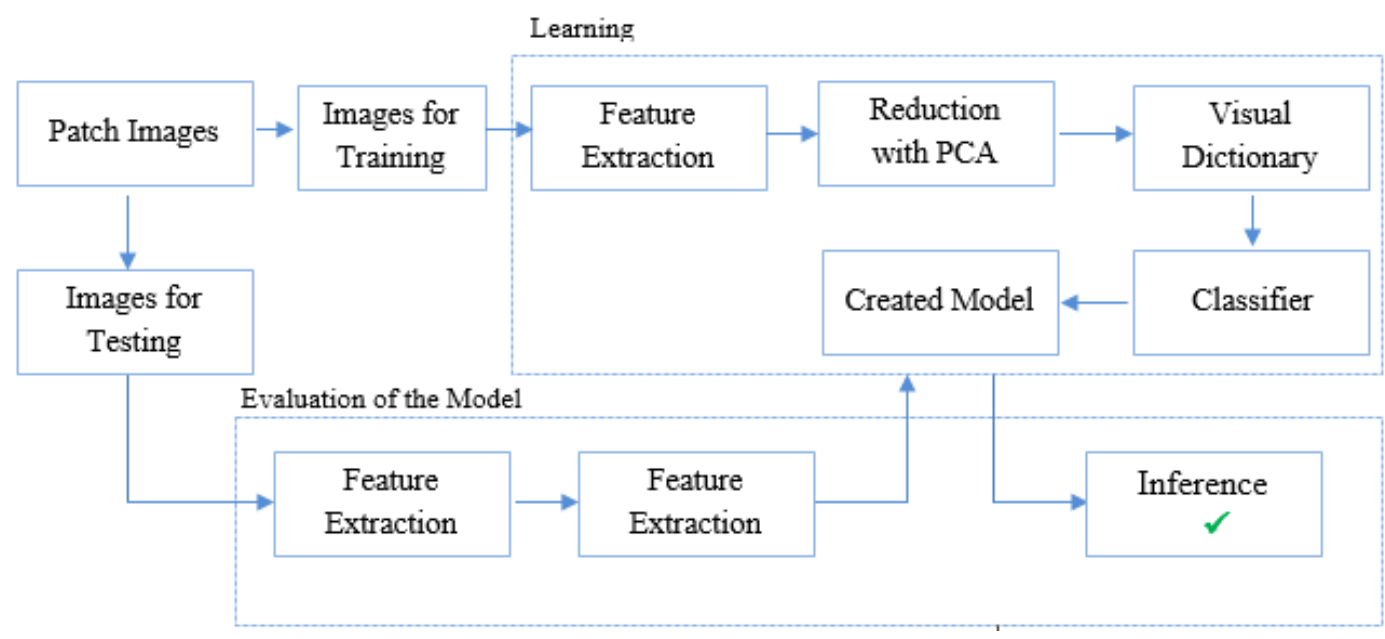

Fig 1: The flowchart of our methodology

For training the classifier, we fed it with feature vectors calculated using the training image examples called pathological and non-pathological. To better assess the performance of these various classification schemes on our dataset, we employed 5-fold cross-validation for multiple runs, and then calculated the mean accuracy of classification over all runs. The result of this classification is shown in Table 1. Successful classifications, as seen in each testing process, have led to the completion of the final stage of the model. Besides, Table 2 shows the Sensitivity, Specificity and Accuracy values in order to evaluate the classification performance for each test process.

Table 1. Classification evaluation results

\begin{tabular}{|c|c|c|c|c|c|c|c|c|c|c|}
\hline & \multicolumn{10}{|c|}{ Classification based codebook-1(with K-Means) } \\
\hline & \multicolumn{2}{|c|}{ Fold 1} & \multicolumn{2}{|c|}{ Fold 2} & \multicolumn{2}{|c|}{ Fold 3} & \multicolumn{2}{|c|}{ Fold 4} & \multicolumn{2}{|c|}{ Fold 5} \\
\hline & $\begin{array}{c}\text { Non- } \\
\text { Exudate }\end{array}$ & Exudate & $\begin{array}{c}\text { Non- } \\
\text { Exudate }\end{array}$ & Exudate & $\begin{array}{c}\text { Non- } \\
\text { Exudate }\end{array}$ & Exudate & $\begin{array}{c}\text { Non- } \\
\text { Exudate }\end{array}$ & Exudate & $\begin{array}{c}\text { Non- } \\
\text { Exudate }\end{array}$ & Exudate \\
\hline Non- Exudate & 85.7 & 14.3 & 100.0 & 0.0 & 88.5 & 11.5 & 92.6 & 7.4 & $96.4 \%$ & $3.6 \%$ \\
\hline \multirow[t]{4}{*}{ Exudate } & 12.9 & 87.1 & 10.0 & 90.0 & 6.1 & 93.9 & 9.4 & 90.6 & $0.0 \%$ & $100.0 \%$ \\
\hline & \multicolumn{10}{|c|}{ Classification based codebook-2 (with DoG) } \\
\hline & \multicolumn{2}{|c|}{ Fold 1} & \multicolumn{2}{|c|}{\begin{tabular}{|l|} 
Fold 2 \\
\end{tabular}} & \multicolumn{2}{|c|}{ Fold 3} & \multicolumn{2}{|c|}{ Fold 4} & \multicolumn{2}{|c|}{ Fold 5} \\
\hline & $\begin{array}{c}\text { Non- } \\
\text { Exudate }\end{array}$ & Exudate & $\begin{array}{c}\text { Non- } \\
\text { Exudate }\end{array}$ & Exudate & $\begin{array}{c}\text { Non- } \\
\text { Exudate }\end{array}$ & Exudate & $\begin{array}{c}\text { Non- } \\
\text { Exudate }\end{array}$ & Exudate & $\begin{array}{c}\text { Non- } \\
\text { Exudate }\end{array}$ & Exudate \\
\hline Non- Exudate & 96.4 & 3.6 & 94.9 & 5.1 & 88.5 & 11.5 & 100.0 & 0.0 & 92.9 & 7.1 \\
\hline Exudate & 3.2 & 96.8 & 0.0 & 100.0 & 6.1 & 93.9 & 9.4 & 90.6 & 6.5 & 93.5 \\
\hline
\end{tabular}


Table 2. Classification evaluation results

\begin{tabular}{|c|c|c|c|c|c|c|c|c|c|c|}
\hline & \multicolumn{4}{|c|}{ Model 1 (based codebook-1) } & \multicolumn{5}{c|}{ Model 2 (based codebook-2) } \\
\cline { 2 - 12 } & Fold 1 & Fold 2 & Fold 3 & Fold 4 & Fold 5 & Fold 1 & Fold 2 & Fold 3 & Fold 4 & Fold 5 \\
\hline SN & 86.21 & 95.12 & 92.30 & 89.28 & 99.99 & 96.55 & 99.99 & 92.30 & 90.0 & 92.85 \\
\hline SP & 87.09 & 99.99 & 91.18 & 93.75 & 96.96 & 96.77 & 91.30 & 91.17 & 99.99 & 93.75 \\
\hline Accuracy & 86.66 & 96.66 & 91.66 & 91.66 & 98.33 & 96.66 & 96.66 & 91.66 & 95.0 & 93.33 \\
\hline
\end{tabular}

Thus, the process of modeling of the system, required for the analysis of the new retina, was completed. Our study has produced 2 models based on two codebooks. The accuracy of the created models were tested on 8 retinal fundus images, with DR identified and randomly selected, with validation approach in order to evaluate the success of the models. As seen in Figure 2, to analysis a new retinal image, the workflow is as follows:
- Detection the PoIs of the image using ORB keypoint algorithm,

- After finding the PoIs, describing the image patches using local features algorithms,

- Sending the found values to the classifier which will classify an image as pathological or nonpathological.

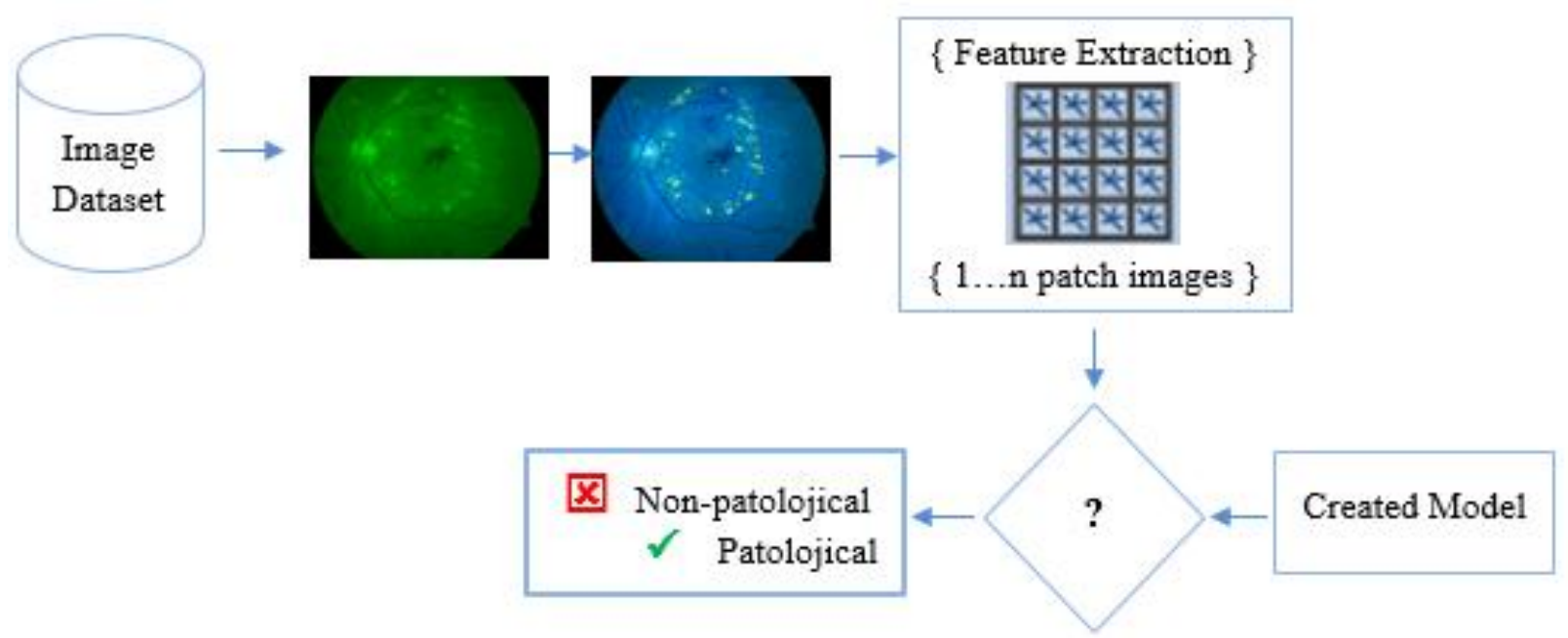

Fig 2. Diagram of the analysis of a new retinal image

Classification data of each retina are summarized in Table 2. For example, actually while there are 6 pathological and 7 non-pathological regions in retina-01, it is observed confusion matrix on which there are correctly classified 6 pathological and 6 non-pathological regions based first codebook, and successful classification rate is $99.98 \%$ for this retinal fundus image. There are correctly classified 6 pathological and 4 nonpathological region based second codebook, and successful classification rate is $76.92 \%$ for this retinal fundus image. When examined classification results concerning all retinas, it is seen that the created model by first codebook is more successful than the second.

Table 2. Classification evaluation results of the new retinal fundus images

\begin{tabular}{|c|c|c|c|c|c|c|c|c|}
\hline \multicolumn{9}{|c|}{ a) $\quad$ Created model with KMeans codebook } \\
\hline & \multicolumn{2}{|c|}{ Retina 01} & \multicolumn{2}{|c|}{ Retina 02} & \multicolumn{2}{|c|}{ Retina 03} & \multicolumn{2}{|c|}{ Retina 04} \\
\hline & $\begin{array}{c}\text { Non- } \\
\text { Exudate }\end{array}$ & Exudate & $\begin{array}{c}\text { Non- } \\
\text { Exudate }\end{array}$ & Exudate & $\begin{array}{c}\text { Non- } \\
\text { Exudate }\end{array}$ & Exudate & $\begin{array}{c}\text { Non- } \\
\text { Exudate }\end{array}$ & Exudate \\
\hline Non- Exudate & 6 & 1 & 0 & 3 & 0 & 0 & 0 & 4 \\
\hline \multirow[t]{5}{*}{ Exudate } & 0 & 6 & 6 & 82 & 1 & 29 & 7 & 65 \\
\hline & Accuracy & 99.98 & Accuracy & 90.10 & Accuracy & 96.66 & Accuracy & 85.52 \\
\hline & & & & & & & & \\
\hline & \multicolumn{2}{|c|}{ Retina 05} & \multicolumn{2}{|c|}{ Retina 06} & \multicolumn{2}{|c|}{ Retina 07} & \multicolumn{2}{|c|}{ Retina 08} \\
\hline & $\begin{array}{c}\text { Non- } \\
\text { Exudate }\end{array}$ & Exudate & $\begin{array}{c}\text { Non- } \\
\text { Exudate }\end{array}$ & Exudate & $\begin{array}{c}\text { Non- } \\
\text { Exudate }\end{array}$ & Exudate & $\begin{array}{c}\text { Non- } \\
\text { Exudate }\end{array}$ & Exudate \\
\hline Non- Exudate & 1 & 1 & 1 & 1 & 0 & 1 & 1 & 0 \\
\hline \multirow[t]{2}{*}{ Exudate } & 5 & 29 & 1 & 9 & 4 & 13 & 18 & 69 \\
\hline & Accuracy & 83.33 & Accuracy & 83.33 & Accuracy & 72.22 & Accuracy & 79.54 \\
\hline
\end{tabular}




\begin{tabular}{|c|c|c|c|c|c|c|c|c|}
\hline \multicolumn{9}{|c|}{ b) $\quad$ Created model with DoG codebook } \\
\hline & \multicolumn{2}{|c|}{ Retina 01} & \multicolumn{2}{|c|}{ Retina 02} & \multicolumn{2}{|c|}{ Retina 03} & \multicolumn{2}{|c|}{ Retina 04} \\
\hline & $\begin{array}{c}\text { Non- } \\
\text { Exudate }\end{array}$ & Exudate & $\begin{array}{c}\text { Non- } \\
\text { Exudate }\end{array}$ & Exudate & $\begin{array}{c}\text { Non- } \\
\text { Exudate }\end{array}$ & Exudate & $\begin{array}{c}\text { Non- } \\
\text { Exudate }\end{array}$ & Exudate \\
\hline Non- Exudate & 4 & 3 & 0 & 3 & 0 & 0 & 0 & 4 \\
\hline \multirow[t]{4}{*}{ Exudate } & 0 & 6 & 0 & 88 & 4 & 26 & 13 & 59 \\
\hline & Accuracy & 76.92 & Accuracy & 96.70 & Accuracy & 86.66 & Accuracy & 77.63 \\
\hline & \multicolumn{2}{|c|}{ Retina 05} & \multicolumn{2}{|c|}{ Retina 06} & \multicolumn{2}{|c|}{ Retina 07} & \multicolumn{2}{|c|}{ Retina 08} \\
\hline & $\begin{array}{c}\text { Non- } \\
\text { Exudate }\end{array}$ & Exudate & $\begin{array}{c}\text { Non- } \\
\text { Exudate }\end{array}$ & Exudate & $\begin{array}{c}\text { Non- } \\
\text { Exudate }\end{array}$ & Exudate & $\begin{array}{c}\text { Non- } \\
\text { Exudate }\end{array}$ & Exudate \\
\hline Non- Exudate & 1 & 1 & 0 & 2 & 0 & 1 & 0 & 1 \\
\hline Exudate & 1 & 33 & 5 & 5 & 1 & 16 & 15 & 72 \\
\hline & Accuracy & 94.44 & Accuracy & 41.67 & Accuracy & 88.88 & Accuracy & 81.81 \\
\hline
\end{tabular}

\section{DISCUSSION AND FUTURE RESEARCH}

As a future work we propose practice with new feature vectors and distinctive attributes using local descriptors algorithms and feature selection algorithms. The use of new descriptors can increase the efficiency of classification and consequently the result of classification. Beside, we consider working with other clustering algorithms such as hierarchal clustering for visual dictionary. Another future work proposal is the identification of the other retinal pathologies such as hemorrhage, red spots.

\section{CONCLUSIONS}

Both eyes are affected usually by diabetic retinopathy. In the disease's early stages, people having diabetic retinopathy often do not realize changes in their vision. But as it progresses, it becomes impossible not to notice the vision loss in many cases cannot be reversed. One of the most successful means for fighting DR in developed and developing countries continues to be its early diagnosis through the analysis of retinal fundus images of the human retina. Hard exudates are deposits of cholesterol or other fats from the blood that have leaked into the retina.

Image processing constitutes the beginning of processes for strong sense from image. After, visual information is consulted for image retrieval. Visual information represent image as the best possible way by distinguishing between images that different categories. In this direction, we have used a visual dictionary approach for detecting hard exudate lesions in retinal fundus images. Visual dictionary is an elegant method to learn and represent important features of any patch image. It allows us to classify whether retinal fundus image is normal or HE candidate. After, we created models with these visual dictionaries. Consequently, we showed the effectiveness of our contribution on new retinal fundus images using these models.

\section{ACKNOWLEDGMENTS}

We thank the DIARETDB1 project for making their images publicly available.

\section{REFERENCES}

[1] Bernardes, R., Serranho, P., and Lobo, C. 2011. Digital ocular fundus imaging: A review. Ophthalmologica, 226(4) (Oct. 2011), 161-181.

[2] Mohamed, Q., Gillies, M.C. and Wong, T.Y. 2007. Management of diabetic retinopathy: A systematic review, The Journal of the American Medical Association, JAWA, 298(8), 902-916.

[3] Venkatesan, R., Chandakkar, P., Li, B., Li, H.K. 2012. Classification of Diabetic Retinopathy Images Using Multi-Class Multiple-Instance Learning Based on Color Correlogram Features, Conf Proc IEEE Eng Med Biol Soc.

[4] Kauppi, T., Kalesnykiene, V., Kamarainen, J.K., Lensu, L., Sorri, I., Raninen, A., Voutilainen, R., Pietila, J., Kalviainen, H., Uusitalo, H. 2007. Diaretdb1 Diabetic Retinopathy Database and Evaluation Protocol, Proceedings of the Medical Image Understanding and Analysis

[5] Chen, X., Bu, W., Wu, X., Dai, B., Teng, Y. 2012. A novel method for automatic Hard Exudates detection in color retinal images, International Conference on Machine Learning and Cybernetics (ICMLC)

[6] Eadgahi, M.G.F., Pourreza, H. 2012. Localization of hard exudates in retinal fundus image by mathematical morphology operations, 2nd International Conference on Computer and Knowledge Engineering (ICCKE)

[7] Garcia, M., Valverde, C., Lopez, M.I., Poza, J., Hornero, R. 2013. Comparison of logistic regression and neural network classifiers in the detection of hard exudates in retinal images, "Engineering in Medicine and Biology Society (EMBC), 35th Annual International Conference of the IEEE"

[8] Fang, G., Yang, N., Lu, H., Li, K. 2010. Automatic segmentation of hard exudates in fundus images based on boosted soft segmentation, International Conference on Intelligent Control and Information Processing (ICICIP)

[9] Hsu, W., Pallawala, P.M.D., Mong L.L., Kah-Guan A.E. 2001. The role of domain knowledge in the detection of retinal hard exudates, Computer Vision and Pattern Recognition, Proceedings of the IEEE Computer Society Conference

[10] Kayal, D. and Banerjee, S. 2014. A new dynamic thresholding based technique for detection of hard exudates in digital retinal fundus image, International Conference on Signal Processing and Integrated Networks (SPIN)

[11] Mishra, A.M., Singh, P.K., Chawla, K.S. 2011. An information geometry based scheme for hard exudate detection in fundus Images, India Conference (INDICON) 
[12] Ranamuka, N.G., Meegama, R.G.N. 2013. Detection of hard exudates from diabetic retinopathy images using fuzzy logic, Image Processing, IET 7(2), 121-130.

[13] Sánchez, C.I., Niemeijer, M., Suttorp Schulten, M.S.A., Abràmoff, M., Van Ginneken, B. 2010. Improving hard exudate detection in retinal images through a combination of local and contextual information, International Symposium on Biomedical Imaging: From Nano to Macro

[14] Sanchez, C.I., Mayo, A., Garcia, M., Lopez, M.I., Hornero, R. 2006. Automatic Image Processing Algorithm to Detect Hard Exudates based on Mixture Models, 28th Annual International Conference of the IEEE Engineering in Medicine and Biology Society

[15] Tjandrasa, H., Putra, R.E., Wijaya, A.Y., Arieshanti, I. 2013. Classification of non-proliferative diabetic retinopathy based on hard exudates using soft margin SVM, IEEE International Conference on Control System, Computing and Engineering (ICCSCE)

[16] Xu, L. and Luo S. 2009. Support vector machine based method for identifying hard exudates in retinal images, IEEE Youth Conference on Information Computing and Telecommunication

[17] Akyol, K., Bayır, Ş., Şen, B., Kaya, H. 2015. Automated Detection of Optic Disc in Retinal Fundus Images using Gabor Filter Kernels, 5th World Conference on Innovation And Computer Sciences

[18] Kaya H., Çavuşoğlu A., Çakmak H.B., Şen B., Delen D. 2014. Determining the Correct Diagnosis and Appropriate Treatment Method on Keratoconus: a 3D Decision Support Application, Global Conference on Healthcare Systems Engineering (GCHSE)

[19] Yang J., Jiang Y.G., Hauptmann A.G., Ngo C.W. 2007. Evaluating bag-of-visual-words representations in scene classification, Proceeding MIR '07 Proceedings of the international workshop on Workshop on multimedia information retrieval
[20] Mikolajczyk, K., Schmid C. 2005. A performance evaluation of local descriptors, IEEE Transactions on Pattern Analysis and Machine Intelligence, 27(10) (Oct 2005), 1615-1630.

[21] Rublee, E., Rabaud, V., Konolige, K., Bradski, G. 2011 "ORB: an efficient alternative to SIFT or SURF", IEEE International Conference on Computer Vision (ICCV)

[22] MacQueen, J. 1967 Some methods for classification and analysis of multivariate observations. University of California Press.

[23] Krig, S. 2014 Computer Vision Metrics: Survey, Taxonomy, and Analysis. Apress Open.

[24] Haykin, S. 2001 Neural Networks: A Comprehensive Foundation. Prentice Hall.

[25] Umesh K. K. and Suresha 2012. Web Image Retrieval Using Visual Dictionary, International Journal on Web Service Computing (IJWSC), 3(3) (September 2012), 77 84

[26] Breiman L. 2001 Random forests. Machine Learning. University of California

[27] Bosch, A., Zisserman, A., Muoz, X. 2007. Image Classification using Random Forests and Ferns, IEEE 11th International Conference on Computer Vision.

[28] Parikh, R., Mathai, A., Parikh, S., Sekhar G.C., Thomas, R. 2008. Understanding and using sensitivity, specificity and predictive values, Indian J Ophthalmol, 56(1) (JanFeb-2008), 45-50.

[29] Lichode, R.V., An approach to Enhance Automatic Diagnosis of Diabetic Retinopathy and Classification by Hybrid Multilayer Feed forward Neural Networks by Genetic Algorithm, International Journal of Science, Engineering and Technology Research (IJSETR), 4(4) (April 2015), $2278-7798$. 